\title{
Evaluating the Safety and Efficacy of Nivolumab in Patients with Advanced Hepatocellular Carcinoma: Evidence to Date
}

This article was published in the following Dove Press journal: OncoTargets and Therapy

\author{
Sri Harsha Tella' \\ Amit Mahipal (iD) ${ }^{2}$ \\ Anuhya Kommalapati ${ }^{3}$ \\ Zhaohui Jin (iD) ${ }^{2}$ \\ 'Department of Internal Medicine, \\ University of South Carolina School of \\ Medicine, Columbia, SC, USA; \\ ${ }^{2}$ Department of Medical Oncology, Mayo \\ Clinic, Rochester, MN, USA; \\ ${ }^{3}$ Department of Hematology and Medical \\ Oncology, H. Lee Moffitt Cancer Center, \\ Tampa, FL, USA
}

\begin{abstract}
Hepatocellular carcinoma (HCC) is the most common primary liver cancer with a dismal prognosis, especially when diagnosed at advanced stages. Surgical resection of the primary lesion, liver-directed therapies, and orthotropic liver transplantation are employed in localized disease depending upon the clinical status, underlying liver function, the size, and location of the liver lesions. Systemic therapy plays a critical role in the management of advanced HCC. Sorafenib had remained as the only United States Food and Drug Administration (US-FDA)-approved systemic therapeutic agent for approximately a decade since its approval in 2007, until the advent of immunotherapy and a better understanding of HCC molecular pathogenesis changed the landscape of advanced HCC management. Lenvatinib was approved as an alternative first-line agent, whereas regorafenib, nivolumab, pembrolizumab, ramucirumab, and cabozantinib were approved as second-line agents for HCC patients who could not tolerate or whose disease progressed on sorafenib. Nivolumab and pembrolizumab are the two immunotherapeutic agents that were conditionally approved by the US-FDA based on the encouraging results in Phase I/II trials. This review discusses the potential role of immunotherapy in advanced HCC with a special focus on nivolumab.
\end{abstract}

Keywords: liver cancer, immunotherapy, nivolumab, lenvatinib, sorafenib

\section{Introduction}

Hepatocellular carcinoma (HCC) is the most common primary liver cancer with the estimated global burden of 22 million over the next two decades. ${ }^{1}$ The majority of the HCC cases are reported in the Asia-Pacific region followed by the sub-Saharan African region. $^{2}$ The high incidence of HCC in these regions is attributed to chronic inflammation resulting from viral or parasitic infections, alcohol, tobacco, and aflatoxin exposure. ${ }^{3}$ While the vaccination against hepatitis $\mathrm{B}$ virus has led to a decreased incidence of $\mathrm{HCC}$ in the Eastern world, rising rates of metabolic issues such as obesity and non-alcoholic fatty liver disease (NAFLD) contributed to an increasing incidence of HCC in the Western world. ${ }^{4-6}$ Chronic inflammation due to infectious and metabolic risk factors leads to hepatic tissue transformation and remodeling that ultimately result in liver cirrhosis. Most patients have cirrhosis at the time of the initial diagnosis of HCC. This chronic inflammatory status and the hepatic tissue inherent "immune escape" mechanism potentiate the progression of cirrhosis to $\mathrm{HCC}^{7}$

The patient's clinical status, Child-Pugh score, Barcelona-Clinic liver cancer system score, and the disease extent determine the appropriate therapeutic options in HCC.
Correspondence: Zhaohui jin

Department of Medical Oncology, Mayo

Clinic, 200 Ist Street SW, Rochester, MN

55905, USA

Tel + I507-293-0487

Fax + | 507-284-1803

Email Jin.Zhaohui@mayo.edu 
Surgical resection of the primary tumor, liver-directed therapies such as trans-arterial chemoembolization (TACE), radiofrequency ablation (RFE), and stereotactic body radiation therapy (SBRT) are employed as first-line treatment options in the localized disease. Orthotropic liver transplantation is preferred in Child-Pugh class $\mathrm{B}$ and $\mathrm{C}$ patients who have solitary tumor $<5 \mathrm{~cm}, 3$ individual tumors with each $\leq 3$ $\mathrm{cm}$ in maximum diameter without extra-hepatic (including regional nodal disease) or vascular extension (the Milan criteria).

Systemic therapy is a key treatment option in patients with portal vein involvement, extra-hepatic extension, and disease recurrence after surgery and in those whose disease progressed after liver-directed therapy. After the encouraging results of sorafenib with a median overall survival of 10.7 months vs 7.9 months as compared to placebo (SHARP trial), ${ }^{8}$ there was a sad saga of almost a decade where no systemic therapeutic agent showed overall survival benefits. Regorafenib, an anti-angiogenic multi-kinase inhibitor, was approved by the United States Food and Drug Administration (US-FDA) in 2017 in advanced HCC patients as a second-line agent after sorafenib. ${ }^{9}$ Later, in 2018, US-FDA approved lenvatinib as an alternative first-line systemic therapeutic agent after lenvatinib showed non-inferiority to sorafenib on overall survival in a Phase III trial. ${ }^{10}$ Another small molecule multi-kinase inhibitor, cabozantinib showed improved overall and progression-free survival as compared to that of placebo in advanced HCC patients who progressed on sorafenib, which resulted in its approval by the FDA. ${ }^{11}$ Most recently, a monoclonal antibody ramucirumab targeting Vascular Endothelial Growth Factor Receptor 2 (VEGFR2) was approved by the US-FDA in a subset of patients with elevated alfa-fetoprotein levels (>400 ng/mL) based on the overall survival benefits compared with placebo in the second-line setting. ${ }^{12}$ Though immunotherapy with cytokines (interferon alpha-2b, interleukin-12) ${ }^{13,14}$ did not yield in encouraging results, recent Phase I/II clinical trials involving checkpoint inhibitors such as nivolumab and pembrolizumab demonstrated potential benefits of immunotherapy in advanced HCC. ${ }^{15,16}$ In addition, immunotherapy is currently being evaluated in combination with liver-directed therapies and as adjuvant therapy for HCC. Ongoing translational and clinical research will hopefully provide us a better understanding of tumor markers, genetic aberrations, and other factors that determine the immunotherapy response in HCC. This review summarizes the HCC immune escape mechanism and the role of checkpoint inhibition in advanced HCC and discusses the efficacy and safety of nivolumab in advanced HCC.

\section{Inherent Immune Tolerance in Hepatic Tissue and Its Role in HCC Tumorigenesis}

As hepatic tissue acts as a first barrier for toxins and antigens from the gastrointestinal tract, it has intrinsic tolerance and immune escape mechanisms to prevent any auto-immune destruction from cytotoxic T-cells. Inhibitory cytokines such as interleukins-4, 5, 8, 10 and tumor growth factor (TGF)- $\beta$ play a significant role in achieving this immunosuppressive effect in the liver tissue. ${ }^{17} \mathrm{CD} 4+\mathrm{T}$-cell activation is also limited due to decreased expression of CD80 and CD86 surface molecules on the antigen-presenting cells. ${ }^{18}$ Furthermore, suppression of NK-T cells and upregulation of myeloid-derived suppressor cells (MDSCs), regulatory dendritic cells, regulatory T (Treg) cells, and immune checkpoint pathways were demonstrated. ${ }^{19-21}$ Indeed, studies have shown the association of increased Treg numbers with poor prognosis. ${ }^{22}$ MDSCs potentiate the HCC tumorigenesis due to their pro-angiogenic activity and immune-suppressive effects by inhibiting NK- and effector T-cells. ${ }^{23}$

Despite this immune escape micro-environment in the liver tissue, activation of CD8+ T-cell immunity was still seen after liver-directed therapies. Examination of blood from HCC patients showed CD8+ T-cell immune responses against the tumor-specific antigen, NY-ESO- $1 .{ }^{24}$ It was found that the presence of $\mathrm{CD} 8+\mathrm{T}$ cells in $>50 \%$ of the HCC tumors analyzed was correlated with progression-free survival, which may serve as the basis for immune-targeted therapies in $\mathrm{HCC}^{25}$

\section{Checkpoint Inhibition as Potential Target in HCC}

Checkpoint inhibitor pathway plays a significant role in immune tolerance environment in the hepatic tissue. Immune checkpoints normally prevent uncontrolled autologous immunity by suppressing the $\mathrm{T}$ cell activation. Overexpression of immune checkpoint molecules by various types of cells, including cancer cells, is an important mechanism for tumor cells to evade the immune surveillance. The PD-1 ligands (PD-L1/L2) that are present on Kupffer cells and other antigen-presenting cells in the liver tissue competitively bind to their respective receptors (PD-1) on T-cells. ${ }^{26}$ This competitive inhibition blocks the CD28 stimulatory activity on the T-cells and potentiates their exhaustion and 
cell death. ${ }^{7}$ Preclinical studies have shown that chronic inflammation of liver tissue induces altered checkpoint inhibitor expression on dendritic cells and altered T-cell pools in the hepatic tissue, thereby inhibiting the immune attack on the affected hepatocytes. ${ }^{27}$ In addition, the association of upregulated PD-1 expression with the progression of cirrhosis to HCC has been reported. ${ }^{28,29}$ This enhanced expression of programmed death ligand 1/2 (PD-L1/L2), especially in chronic inflammatory states, induces T-cell apoptosis potentiating the immune tolerance. ${ }^{30}$ Since the advent of nivolumab and pembrolizumab (both are anti-PD-1 inhibitors) in cutaneous melanoma, immunotherapy targeting the checkpoint pathway has revolutionized the landscape of cancer medicine. Given this association of augmented PD-1/PDL1 expression in chronic inflammatory states, theoretically, checkpoint inhibitors are being evaluated as promising agents in advanced HCC. These immune checkpoint inhibitors act by inducing cytotoxic T-cell-mediated cancer cell death. Immune checkpoint inhibitors such as tremelimumab, durvalumab, atezolizumab, nivolumab, and pembrolizumab are currently being evaluated in advanced HCC. Table 1 summarizes the key clinical trials of checkpoint inhibitors in advanced HCC. ${ }^{15,16,31-34}$

Nivolumab, a human immunoglobulin G4 monoclonal antibody targeting the PD-1 immune checkpoint signaling pathway potentiating the activity of effector T-cells, has shown promising results in Phase I and II clinical studies involving the patients with advanced HCC. ${ }^{15}$ Given the promising results, nivolumab becomes the first immunotherapeutic agent approved (conditionally) by the FDA for the management of HCC. In the following sections, we discuss the efficacy, safety, and future directions of nivolumab in the management of HCC.

\section{Nivolumab as a Monotherapy in the Management of HCC}

With the encouraging results of nivolumab in multiple tumor types including metastatic melanoma, non-smallcell carcinoma of lung, and renal and urothelial cancers, nivolumab was evaluated in a Phase $\mathrm{Ib} / \mathrm{II}$ clinical trial involving advanced HCC patients whose disease progressed on sorafenib or who did not tolerate sorafenib (Checkmate-040 trial-NCT01658878). ${ }^{15}$ The trial included Child-Pugh class A HCC patients who had aspartate aminotransferase (AST) and alanine aminotransferase (ALT) levels $\leq 5 \times$ upper limit of normal, total bilirubin level $\leq 3$ $\mathrm{mg} / \mathrm{dL}$, albumin $\geq 2.8 \mathrm{~g} / \mathrm{dL}$, and platelets $\geq 60 \times 10^{3} / \mu \mathrm{L}$. Patients with a history of immunomodulatory drug exposure, hepatic encephalopathy, and clinically significant ascites were excluded. Among the 262 HCC patients who met the eligibility criteria, 48 were enrolled in the dose-escalation phase (Phase Ib), whereas 214 were included in the dose-expansion cohort. In the dose-escalation phase, $71 \%$ and $40 \%$ of the patients had extrahepatic metastases and extra-vascular invasion, respectively, whereas the corresponding numbers were $67 \%$ and $29 \%$ in the dose-expansion phase. Within these 2 cohorts, patients were further sub-grouped based on the viral etiology - no viral infection, hepatitis C-related HCC, and hepatitis B-related HCC. For patients who had hepatitis

Table I Key Clinical Trials of Immune Checkpoint Inhibitors in Hepatocellular Carcinoma

\begin{tabular}{|c|c|c|c|c|c|c|c|}
\hline \multirow[t]{2}{*}{ Study } & \multirow{2}{*}{$\begin{array}{l}\text { Type of } \\
\text { Study }\end{array}$} & \multirow{2}{*}{$\begin{array}{l}\text { Check-Point } \\
\text { Inhibitor } \\
\text { Evaluated }\end{array}$} & \multicolumn{5}{|c|}{ Significance/Outcome } \\
\hline & & & $\begin{array}{l}\text { Complete } \\
\text { Response }\end{array}$ & $\begin{array}{l}\text { Partial } \\
\text { Response }\end{array}$ & $\begin{array}{l}\text { Stable } \\
\text { Disease }\end{array}$ & Overall Survival & $\begin{array}{l}\text { Progression- } \\
\text { Free } \\
\text { Survival }\end{array}$ \\
\hline El-Khoueiry et al $^{15}(n=48)$ & Phase lb & Nivolumab & $4.2 \%$ & $8.3 \%$ & - & 15 months & 3.4 months \\
\hline El-Khoueiry et $\mathrm{al}^{15}(n=2 \mid 4)$ & Phase II & Nivolumab & $1.4 \%$ & $18.2 \%$ & $45 \%$ & $83 \%$ alive at 6 months & 4.I months \\
\hline Zhu et al ${ }^{16}(n=104)$ & Phase II & Pembrolizumab & $1 \%$ & $16 \%$ & $44 \%$ & $54 \%$ alive at I year & 5.1 months \\
\hline Sangro et $\mathrm{al}^{3 \mathrm{I}}(\mathrm{n}=2 \mathrm{I})$ & Phase II & Tremelimumab & $0 \%$ & $17.6 \%$ & $\sim 59 \%$ & 8.2 months & 6.4 months \\
\hline Duffy et $\mathrm{al}^{32}(\mathrm{n}=32)$ & Phase II & $\begin{array}{l}\text { Tremelimumab+ } \\
\text { ablation }\end{array}$ & - & $26.3 \%$ & - & 12.3 months & 7.4 months \\
\hline Wainberg et $\mathrm{al}^{33}(n=40)$ & Phase I/II & Durvalumab & $0 \%$ & $10.3 \%$ & $23 \%$ & 13.2 months & - \\
\hline Kelley et $\mathrm{al}^{34}(\mathrm{n}=40)$ & Phase I/II & $\begin{array}{l}\text { Durvalumab + } \\
\text { tremelimumab }\end{array}$ & $0 \%$ & $15 \%$ & $45 \%$ & - & - \\
\hline
\end{tabular}


B viral etiology, viral count $<100$ with concomitant antiviral treatment was the pre-requisite enrollment criteria. A quarter of patients (12 out of 48) had grade III-IV therapyrelated adverse events that included skin rash (23\%), elevated liver enzymes (21\%), pruritis (19\%), and elevated lipase levels (19\%). Therapy-associated severe adverse events were reported in three patients, which were adrenal insufficiency, pemphigus, and liver disorder.

In the dose-escalation phase, the patients were exposed to intravenous nivolumab with the doses ranging from 0.1 to 10 $\mathrm{mg} / \mathrm{kg}$ every 14 days. A significant number of patients discontinued the therapy with nivolumab (96\%) primarily due to disease progression, whereas $4 \%$ had a complete response per the study protocol. The overall response rate was achieved in $15 \%$ (seven patients), which included three complete and four partial responses with the median interval of response of 17 (95\% confidence interval [CI]: 6-24) months. The majority of the patients (5 out of 7) had an initial response within three months of therapy initiation. While the drug was generally tolerated well, the most common adverse events that occurred in at least $10 \%$ of the study cohort were elevated liver enzymes. Based on the data from the dose-escalation study and nivolumab studies in other malignancy, $3 \mathrm{mg} / \mathrm{kg}$ every 2 weeks dose was chosen for the dose-expansion cohort.

In the dose-expansion phase, nivolumab showed encouraging results in all the sub-groups as detailed in Table 2. Among the 214 patients that received nivolumab ( $3 \mathrm{mg} / \mathrm{kg}$ every 2 weeks), the overall response was seen in $20 \%$ by modified response evaluation criteria in solid tumors (RECIST). A retrospective analysis of the PD-L1 membrane expression in $81 \%(\mathrm{n}=174)$ patients in dose-expansion phase showed that $80 \%(\mathrm{n}=140)$ and $20 \%(\mathrm{n}=34)$ of patients had $<1 \%$ and at least $1 \%$ of PD-L 1 membrane expression, respectively. The corresponding objective response rates were $19 \%$ $(n=26 / 140)$ and $26 \%(n=9 / 34)$ in the patients with $<1 \%$ and at least $1 \%$ PD-L1 expression, respectively. Similar to that of the dose-escalation phase, nivolumab had an acceptable safety and tolerability profile irrespective of the virus status. ${ }^{15}$ Grade III/IV therapy-related adverse events and severe adverse events were seen in $19 \%$ and $4 \%$ of the study cohort, respectively. Grade III/IV adverse events occurred in $29 \%$ and $18 \%$ of the patients in sorafenib-naive and sorafenib-experienced groups, respectively.

The efficacy and tolerability of nivolumab were also prospectively analyzed in Child-Pugh Class B patients with HCC $(n=49)$ as a part of the Checkmate- 040 trial. ${ }^{35}$ Among these 49 patients analyzed, vascular invasion and extra-hepatic spread were seen in $57 \%(\mathrm{n}=28)$. After the median follow-up of 7.4 months, $55 \%$ of the patients had disease control while the objective response was seen in $10 \%$. The median overall survival was 7.6 months, which was higher in sorafenibnaive patients ( 9.8 months) as compared to that of the counterparts (7.3 months). Tolerability of nivolumab was similar to that of Child-Pugh A patients: $51 \%$ had therapy-associated adverse events in which $8 \%$ had liver-specific adverse events. Another retrospective case series evaluated the safety profile of nivolumab in 18 Child-Pugh class B HCC patients. ${ }^{36}$ After a median duration of therapy of 2.3 months, complete and partial responses were seen in 2 and 1 patients, respectively. While the majority of the patients $(94 \%, n=13 / 18)$ had grade III/IV adverse events, immunotherapy-associated adverse events were seen in half of the cohort $(50 \%, \mathrm{n}=9 / 18)$. Though the total number of therapy-related adverse events were higher in Child-Pugh class B patients, immune-related adverse events were similar in Child-Pugh class A and class B patients. It is important to note that the rate of serious adverse events seen in this series was similar to that observed with sorafenib in Child-Pugh Class B patients. ${ }^{37,38}$

A single-institution study reported the tolerability of nivolumab in a small cohort of Child-Pugh class $C$ patients with $\operatorname{HCC}(n=3) .{ }^{39}$ The drug had a tolerable safety profile, and one of the three patients had a complete response while on the nivolumab therapy. Adverse events reported were of grade I/II.

While Phase I/II trials show encouraging results of nivolumab therapy in HCC, a randomized Phase III trial (Checkmate 459-NCT02576509) that evaluated nivolumab head to head

Table 2 Summary of Responses Observed in Phase II Trial Evaluating Nivolumab in Advanced HCC (Checkmate-040)

\begin{tabular}{|c|c|c|c|}
\hline Response & No Virus Etiology $(n=56)$ & Hepatitis C Virus HCC $(n=50)$ & $\begin{array}{l}\text { Hepatitis B Virus } \\
\text { HCC }(n=5 I)\end{array}$ \\
\hline Overall response rate & $23 \%(n=13)^{a}$ & $20 \%(n=10)$ & $14 \%(n=7)$ \\
\hline Disease-control rate & $75 \%(n=42)^{b}$ & $66 \%(n=33)$ & $55 \%(n=28)$ \\
\hline 6-month overall survival rate in sorafenib-naive patients & $89 \%(95 \% \text { Cl: } 77-95)^{c}$ & $85 \%$ (95\% Cl: $72-93)$ & $84 \%$ (95\% Cl: 7I-92) \\
\hline
\end{tabular}

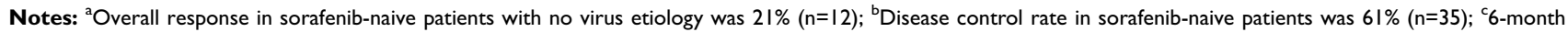
overall survival in sorafenib-exposed patients: $75 \%(95 \% \mathrm{Cl}: 62-85 \%)$; Median overall survival in sorafenib-exposed patients: 13.2 months. Data from ElKhoueiry AB, Sangro B, Yau T, et al. ${ }^{15}$ 
with sorafenib failed to meet the primary end point of improved overall survival in nivolumab cohort $(\mathrm{HR}=0.85$ [95\% CI: $0.72-1.02] ; p=0.07) .{ }^{40}$ However, there seemed to be a trend towards the improved overall survival, as per the press release. A detailed analysis of the study results is not yet available, which will hopefully shed light on the place of nivolumab in advanced HCC armamentarium. More studies with a large cohort of Child-Pugh Class B and C patients are needed to determine the role of nivolumab in the real-world context. However, enrollment of Child-Pugh class B and C patients in randomized clinical trials is a challenge, which may be addressed by initiating multi-institutional, national and international collaborations.

\section{Nivolumab: As a Combination Therapy in Advanced HCC}

Nivolumab was evaluated in combination with ipilimumab in 148 advanced HCC patients whose disease progressed on sorafenib or who discontinued sorafenib due to intolerance in an experimental arm of the Checkmate 040 studyNCT01658878. ${ }^{41}$ These patients were randomized to three combination doses of nivolumab and ipilimumab: nivolumab $1 \mathrm{mg} / \mathrm{kg}+$ ipilimumab $3 \mathrm{mg} / \mathrm{kg}$ every 3 weeks (arm $\mathrm{A}, \mathrm{n}=50$ ); nivolumab $3 \mathrm{mg} / \mathrm{kg}+$ ipilimumab $1 \mathrm{mg} / \mathrm{kg}$ every 3 weeks followed by nivolumab $240 \mathrm{mg}$ every 2 weeks ( $\operatorname{arm} \mathrm{B}, \mathrm{n}=49$ ); or nivolumab $3 \mathrm{mg} / \mathrm{kg}$ every 2 weeks + ipilimumab $1 \mathrm{mg} / \mathrm{kg}$ every 6 weeks ( $\operatorname{arm} \mathrm{C}, \mathrm{n}=49$ ). Both arm A and arm B were followed by nivolumab $240 \mathrm{mg}$ every 2 weeks. A total of $31 \%$ of the study cohort had an overall response with 24-month overall survival rate of $40 \%$. The patients in arm A had a median overall survival of 24 months in which $8 \%, 24 \%$, and $18 \%$ had complete, partial response, and stable disease, respectively. It is important to note that the objective response rate of the combination therapy of nivolumab and ipilimumab was twice $(31 \%)$ than that of nivolumab monotherapy (14\%). The combination therapy is well tolerated with grade IIIIV adverse events being reported in $37 \%$ of the cohort, whereas $5 \%$ discontinued the combination regimen due to the adverse events. Other ongoing checkpoint inhibitor combination clinical trials involving nivolumab in advanced HCC are summarized in Table 3 . The combination of nivolumab and ipilimumab was also evaluated in the perioperative period in the patients with resectable HCC. $^{42}$ Interim analysis of 14 enrolled patients showed a pathologic complete response of $29 \%$, and no delays in surgical resection were seen. Further information on a large cohort of patients will help to determine the role of checkpoint inhibitors in neoadjuvant and adjuvant settings. Nivolumab and other checkpoint inhibitors are also being evaluated in combination with liver-directed therapies. Selective ongoing clinical trials that evaluate the

Table 3 Selective Ongoing Clinical Trials of Combination of Nivolumab and Other Immune- and Targeted-Therapy Agents in Advanced Hepatocellular Carcinoma

\begin{tabular}{|c|c|c|}
\hline Targeted Pathways & Agents Evaluated & $\begin{array}{l}\text { Clinical Trial } \\
\text { Registration } \\
\text { Number }\end{array}$ \\
\hline CTLA-4+PD-I & Nivolumab + ipilimumab & $\begin{array}{l}\text { NCT03203304 } \\
\text { NCT03222076 }\end{array}$ \\
\hline $\begin{array}{l}\text { GM-CSF-armed } \\
\text { oncolytic virus + PD-I }\end{array}$ & PexaVec+ nivolumab & NCT0307I094 \\
\hline PD-I+ tyrosine kinase inhibitor & $\begin{array}{l}\text { Nivolumab + cabozantinib } \\
\text { Nivolumab + lenvatinib } \\
\text { Nivolumab + sorafenib }\end{array}$ & $\begin{array}{l}\text { NCT03299946 } \\
\text { NCT034I8922 } \\
\text { NCT0343989I }\end{array}$ \\
\hline PD-I+ anti-VEGF agents & Nivolumab + bevacizumab & NCT03382886 \\
\hline PD-I + TGFbeta inhibitor & Nivolumab + galusertinib & NCT02423343 \\
\hline $\begin{array}{l}\text { CTLA-4, PD-I, anti-OX40 } \\
\text { antibody }\end{array}$ & $\begin{array}{l}\text { INCAGN0I949 + ipilimumab vs INCAGN01949 + nivolumab vs INCAGN0I949 + } \\
\text { nivolumab + ipilimumab } \\
\text { INCAGN0I949 + nivolumab vs INCAGN01949 + ipilimumab vs INCAGN01949 + } \\
\text { nivolumab + ipilimumab }\end{array}$ & NCT0324II73 \\
\hline
\end{tabular}

Abbreviations: CTLA4, cytotoxic T-lymphocyte-associated protein 4; PD-I, programmed cell death protein-I; GM-CSF, granulocyte monocyte-colony stimulating factor; VEGF, vascular endothelial growth factor. 
combination of nivolumab with liver-directed therapies are summarized in Table 4.

\section{Future Directions of Nivolumab and Other Checkpoint Inhibitor Therapy in Advanced HCC}

The advent of checkpoint inhibitors has created some hope for immunotherapy in the management of HCC, especially in the last 5 years. Though these checkpoint inhibitors showed encouraging results in Phase I/II clinical trials, a Phase III clinical trial that evaluated the role of pembrolizumab (KEYNOTE-240) in advanced HCC patients who had prior exposure to sorafenib in comparison to best supportive care failed to meet the co-primary end-points of overall and progression-free survival, challenging the role of checkpoint inhibitors in the dismal cancer. ${ }^{43}$ However, it is important to note that there was a trend favoring pembrolizumab with longer median overall survival ( $\mathrm{HR}=0.78 ; \mathrm{CI}: 0.61-0.99)$ and progression-free survival $(\mathrm{HR}=0.78$; $\mathrm{CI}: 0.61-0.99)$. This has been the case with the nivolumab, too. ${ }^{40}$ Phase I/II trials of nivolumab, as a monotherapy and in combination with ipilimumab, showed the objective response rate of $14 \%$ and $31 \%$, respectively. ${ }^{15,41}$ These study results suggest that a subset of people may benefit from the checkpoint inhibitors. Moreover, patients whose diseases respond seem to have prolonged disease control. Given the heterogeneity of HCC, now the challenge is to identify the subset of advanced HCC patients in whom these agents may benefit the most. Advancements in molecular profiling techniques and a better understanding of tumor biology and markers could help in identifying this subset of patients. For example, Harding et al identified the druggable targets and the

Table 4 Selective Ongoing Clinical Trials of Combination of Nivolumab with Liver-Directed Therapies

\begin{tabular}{|l|l|l|}
\hline Targeted Pathways & Agents Evaluated & $\begin{array}{l}\text { Clinical Trial } \\
\text { Registration } \\
\text { Number }\end{array}$ \\
\hline Radiation + PD-I & Yttrium-90+ nivolumab & $\begin{array}{l}\text { NCT03380I30 } \\
\text { NCT03033446 } \\
\text { NCT02837029 }\end{array}$ \\
\hline Ischemia + PD-I & $\begin{array}{l}\text { TACE + nivolumab } \\
\text { DEB-TACE + nivolumab }\end{array}$ & $\begin{array}{l}\text { NCT03572582 } \\
\text { NCT03I43270 }\end{array}$ \\
\hline
\end{tabular}

Abbreviations: PD-I, programmed cell death protein-I; SBRT, stereotactic body radiation therapy; TACE, Trans-Arterial Chemoembolization; DEB-TACE, Drug-Eluting Bead Trans-Arterial Chemoembolization; CTLA4, cytotoxic T-lymphocyte-associated protein 4; PD-LI, programmed cell death ligand-I; RFA, radiofrequency ablation. resistance mechanisms by utilizing the next-generation sequencing technique, which could potentially determine the response of systemic therapy. ${ }^{44}$ Their study showed that the $\mathrm{HCC}$ tumors harboring Wnt/CTNNB1 mutations were refractory to checkpoint inhibitors. All the study participants harboring Wnt/CTNNB1 $(\mathrm{n}=7)$ and AXIN1 $(n=3)$ mutations had progressive disease with inferior median overall survival as compared to that of the counterparts. On the contrary, encouraging responses with checkpoint inhibitors were seen in the patients whose tumors harbor infiltrating lymphocytes. These studies potentially open the door for precision oncology in HCC by integrating next-generation sequencing to match $\mathrm{HCC}$ patients to immunotherapy. Another challenge is that most of the trials that evaluated the checkpoint inhibitors in advanced HCC included Child-Pugh class A patients. Though practically challenging, more information on the role of these drugs in advanced HCC, especially in Child-Pugh class B and $\mathrm{C}$, would be beneficial.

Another area that needs attention for the use of immunotherapy in advanced HCC is the lack of safety data in patients with the history of orthotopic liver transplantation, due to the concern of transplant rejection, which could lead to fatal liver failure. ${ }^{45}$ The ongoing Phase I clinical trial that is designed to evaluate HBV-specific T cell receptor (HBV/ $\mathrm{TCR}$ ) redirected $\mathrm{T}$ cell therapy in the patients with hepatitis B-related HCC who have undergone orthotropic liver transplantation (NCT02686372) will help us to better understand the tolerability and effectiveness of immunotherapy in this subset of patients. While the case reports mention the successful use of immunotherapy in liver transplant patients, the long-term safety data are lacking. ${ }^{46,47}$

The future of immunotherapy in $\mathrm{HCC}$ might rely on the combination therapy approach: combinations of two checkpoint inhibitors, combinations of checkpoint inhibitors and targeted therapy including VEGFR blockade, combinations of vaccines such as JX-594 (an oncolytic pox virus vaccine), ${ }^{48}$ or a combination of checkpoint inhibitors with liver-directed therapies such as TACE and RFA. Liver-directed therapies alter the local immune environment and tumor antigens are released into the systemic circulation. Thus, adding immune checkpoint inhibitors to these liver-directed therapies helps in potentiating both tumor and systemic immune responses. ${ }^{49}$ Furthermore, agents that deplete regulatory T-cells in the tumors may act synergistically with checkpoint inhibitors. For example, the combination of anti-OX40 (CD134) monoclonal antibody that is known to deplete regulatory 
T-cells, with anti-PD-1 therapy, resulted in promising activity in the tissues that were previously resistant to anti-PD-1 monotherapy. ${ }^{50,51}$ Similar encouraging results were seen in preclinical models that evaluated anti-lymphocyte activation gene-3 (LAG3) antibodies or anti-T-cell immunoglobulin and mucin domain-containing protein 3 (TIM3) antibodies in combination with anti-PD-1/PD-L1 agents. $^{52,53}$ These OX40, LAG3, and TIM3 antibodies act by depleting the regulatory T-cells, thereby preventing the cytotoxic CD8+ exhaustion.

\section{Conclusion}

The advent of immunotherapy, especially checkpoint inhibitors, has revolutionized the landscape of HCC systemic therapy. As we await the full-detailed results of Phase III trial evaluating the nivolumab monotherapy, a number of Phase I/ II/III clinical trials are currently enrolling patients to further analyze the role of immunotherapy including the cellular therapies (NCT03132792, NCT03146234, NCT03198546, NCT02715362, NCT03130712, NCT02959151, and NCT02 905188), vaccines (NCT03071094 and NCT03203005), cytokines (NCT02240433, NCT01246986, NCT02178358, NCT0 2906397, and NCT02423343), either alone or in combinations in large cohort of patients with advanced HCC. Given the heterogenous nature of $\mathrm{HCC}$, integration of technologies such as next-generation sequencing and better knowledge on the tumor markers may hopefully help identify the subset of patients who may potentially benefit from immunotherapy the most.

\section{Disclosure}

The authors report no conflicts of interest in this work.

\section{References}

1. Ghouri YA, Mian I, Rowe JH. Review of hepatocellular carcinoma: epidemiology, etiology, and carcinogenesis. J Carcinog. 2017;16:1. doi:10.4103/jcar.JCar_9_16

2. Akinyemiju T, Abera S, Ahmed M, et al. The burden of primary liver cancer and underlying etiologies from 1990 to 2015 at the global, regional, and national level: results from the global burden of disease study 2015 . JAMA Oncol. 2017;3(12):1683-1691. doi:10.1001/jamaoncol.2017.3055

3. Forner A, Reig M, Bruix J. Hepatocellular carcinoma. Lancet (London, England). 2018;391(10127):1301-1314. doi:10.1016/S0140-6736(18) 30010-2

4. Jemal A, Bray F, Center MM, Ferlay J, Ward E, Forman D. Global cancer statistics. CA: Cancer J Clin. 2011;61(2):69-90.

5. Makarova-Rusher OV, Altekruse SF, McNeel TS, et al. Population attributable fractions of risk factors for hepatocellular carcinoma in the United States. Cancer. 2016;122(11):1757-1765. doi:10.1002/cncr.29971

6. Masuoka HC, Chalasani N. Nonalcoholic fatty liver disease: an emerging threat to obese and diabetic individuals. Ann N Y Acad Sci. 2013;1281:106-122. doi:10.1111/nyas.12016
7. Roth GS, Decaens T. Liver immunotolerance and hepatocellular carcinoma: patho-physiological mechanisms and therapeutic perspectives. Eur J Cancer (Oxford, England: 1990). 2017;87:101-112. doi:10.1016/j.ejca.2017.10.010

8. Llovet JM, Ricci S, Mazzaferro V, et al. Sorafenib in advanced hepatocellular carcinoma. $N$ Engl J Med. 2008;359(4):378-390. doi:10.1056/NEJMoa0708857

9. Bruix J, Qin S, Merle P, et al. Regorafenib for patients with hepatocellular carcinoma who progressed on sorafenib treatment (RESORCE): a randomised, double-blind, placebo-controlled, Phase 3 trial. Lancet (London, England). 2017;389(10064):56-66. doi:10.1016/S0140-6736 (16)32453-9

10. Kudo M, Finn RS, Qin S, et al. Lenvatinib versus sorafenib in firstline treatment of patients with unresectable hepatocellular carcinoma: a randomised phase 3 non-inferiority trial. Lancet (London, England). 2018;391(10126):1163-1173. doi:10.1016/S0140-6736(18)30207-1

11. Abou-Alfa GK, Meyer T, Cheng AL, et al. Cabozantinib in patients with advanced and progressing hepatocellular carcinoma. $N$ Engl J Med. 2018;379(1):54-63. doi:10.1056/NEJMoa1717002

12. Zhu AX, Kang Y-K, Yen C-J, et al. REACH-2: a randomized, double-blind, placebo-controlled phase 3 study of ramucirumab versus placebo as second-line treatment in patients with advanced hepatocellular carcinoma (HCC) and elevated baseline alpha-fetoprotein (AFP) following first-line sorafenib. J Clin Oncol. 2018;36 (15_suppl):4003.

13. Llovet JM, Sala M, Castells L, et al. Randomized controlled trial of interferon treatment for advanced hepatocellular carcinoma. Hepatology (Baltimore, MD). 2000;31(1):54-58. doi:10.1002/hep.510310111

14. Sangro B, Mazzolini G, Ruiz J, et al. Phase I trial of intratumoral injection of an adenovirus encoding interleukin-12 for advanced digestive tumors. J Clin Oncol. 2004;22(8):1389-1397. doi:10.1200/ JCO.2004.04.059

15. El-Khoueiry AB, Sangro B, Yau T, et al. Nivolumab in patients with advanced hepatocellular carcinoma (CheckMate 040): an open-label, noncomparative, phase 1/2 dose escalation and expansion trial. The Lancet. 2017;389(10088):2492-2502. doi:10.1016/S0140-6736(17)31046-2

16. Zhu AX, Finn RS, Edeline J, et al. Pembrolizumab in patients with advanced hepatocellular carcinoma previously treated with sorafenib (KEYNOTE-224): a non-randomised, open-label phase 2 trial. Lancet Oncol. 2018;19(7):940-952. doi:10.1016/S1470-2045(18)30351-6

17. Budhu A, Forgues M, Ye QH, et al. Prediction of venous metastases, recurrence, and prognosis in hepatocellular carcinoma based on a unique immune response signature of the liver microenvironment. Cancer Cell. 2006;10(2):99-111. doi:10.1016/j.ccr.2006.06.016

18. Knolle PA, Germann T, Treichel U, et al. Endotoxin down-regulates $\mathrm{T}$ cell activation by antigen-presenting liver sinusoidal endothelial cells. J Immunol. 1999;162(3):1401-1407.

19. Ilkovitch D, Lopez DM. The liver is a site for tumor-induced myeloidderived suppressor cell accumulation and immunosuppression. Cancer Res. 2009;69(13):5514-5521. doi:10.1158/0008-5472.CAN-08-4625

20. Hoechst B, Ormandy LA, Ballmaier M, et al. A new population of myeloid-derived suppressor cells in hepatocellular carcinoma patients induces CD4(+)CD25(+)Foxp3(+) T cells. Gastroenterology. 2008;135(1):234-243. doi:10.1053/j.gastro.2008.03.020

21. Han Y, Chen Z, Yang Y, et al. Human CD14+ CTLA-4+ regulatory dendritic cells suppress T-cell response by cytotoxic T-lymphocyte antigen-4-dependent IL-10 and indoleamine-2,3-dioxygenase production in hepatocellular carcinoma. Hepatology (Baltimore, Md). 2014;59(2):567-579. doi:10.1002/hep.v59.2

22. Chen KJ, Lin SZ, Zhou L, et al. Selective recruitment of regulatory $\mathrm{T}$ cell through CCR6-CCL20 in hepatocellular carcinoma fosters tumor progression and predicts poor prognosis. PLoS ONE. 2011;6(9):e24671.

23. Hoechst B, Voigtlaender T, Ormandy L, et al. Myeloid derived suppressor cells inhibit natural killer cells in patients with hepatocellular carcinoma via the NKp30 receptor. Hepatology (Baltimore, MD). 2009;50(3):799-807. doi:10.1002/hep.23054 
24. Korangy F, Ormandy LA, Bleck JS, et al. Spontaneous tumor-specific humoral and cellular immune responses to NY-ESO-1 in hepatocellular carcinoma. Clin Cancer Res. 2004;10(13):4332-4341. doi:10.1158/1078-0432.CCR-04-0181

25. Flecken T, Schmidt N, Hild S, et al. Immunodominance and functional alterations of tumor-associated antigen-specific CD8+ T-cell responses in hepatocellular carcinoma. Hepatology (Baltimore, MD). 2014;59(4):1415-1426. doi:10.1002/hep.26731

26. Francisco LM, Salinas VH, Brown KE, et al. PD-L1 regulates the development, maintenance, and function of induced regulatory $\mathrm{T}$ cells. J Exp Med. 2009;206(13):3015-3029. doi:10.1084/jem.20090847

27. Martinet J, Dufeu-Duchesne T, Bruder Costa J, et al. Altered functions of plasmacytoid dendritic cells and reduced cytolytic activity of natural killer cells in patients with chronic HBV infection. Gastroenterology. 2012;143(6):1586-1596.e1588. doi:10.1053/j. gastro.2012.08.046

28. Shi F, Shi M, Zeng Z, et al. PD-1 and PD-L1 upregulation promotes CD8(+) T-cell apoptosis and postoperative recurrence in hepatocellular carcinoma patients. Int $J$ Cancer. 2011;128(4):887-896. doi:10.1002/ijc. 25397

29. Makarova-Rusher OV, Medina-Echeverz J, Duffy AG, Greten TF. The yin and yang of evasion and immune activation in HCC. $J$ Hepatol. 2015;62(6):1420-1429. doi:10.1016/j.jhep.2015.02.038

30. Bottcher JP, Schanz O, Wohlleber D, et al. Liver-primed memory T cells generated under noninflammatory conditions provide anti-infectious immunity. Cell Rep. 2013;3(3):779-795. doi:10.1016/j. celrep.2013.02.008

31. Sangro B, Gomez-Martin C, de la Mata M, et al. A clinical trial of CTLA-4 blockade with tremelimumab in patients with hepatocellular carcinoma and chronic hepatitis C. J Hepatol. 2013;59(1):81-88. doi:10.1016/j.jhep.2013.02.022

32. Duffy AG, Ulahannan SV, Makorova-Rusher O, et al. Tremelimumab in combination with ablation in patients with advanced hepatocellular carcinoma. J Hepatol. 2017;66(3):545-551. doi:10.1016/j.jhep.2016.10.029

33. Wainberg ZA, Segal NH, Jaeger D, et al. Safety and clinical activity of durvalumab monotherapy in patients with hepatocellular carcinoma (HCC). J Clin Oncol. 2017;35(15_suppl):4071. doi:10.1200/ JCO.2017.35.15_suppl.4071

34. Kelley RK, Abou-Alfa GK, Bendell JC, et al. Phase I/II study of durvalumab and tremelimumab in patients with unresectable hepatocellular carcinoma (HCC): phase I safety and efficacy analyses. $J$ Clin Oncol. 2017;35(15_suppl):4073.

35. Kudo M, Matilla A, Santoro A, et al. Checkmate-040: nivolumab (NIVO) in patients (pts) with advanced hepatocellular carcinoma (aHCC) and Child-Pugh B (CPB) status. J Clin Oncol. 2019;37(4_suppl):327.

36. Kambhampati S, Bauer KE, Bracci PM, et al. Nivolumab in patients with advanced hepatocellular carcinoma and Child-Pugh class B cirrhosis: safety and clinical outcomes in a retrospective case series. Cancer. 2019.

37. Abou-Alfa GK, Amadori D, Santoro A, et al. Safety and efficacy of sorafenib in patients with hepatocellular carcinoma (HCC) and ChildPugh A versus B cirrhosis. Gastrointestinal Cancer Res. 2011;4(2):40-44.

38. Marrero JA, Kudo M, Venook AP, et al. Observational registry of sorafenib use in clinical practice across Child-Pugh subgroups: the GIDEON study. J Hepatol. 2016;65(6):1140-1147. doi:10.1016/j. jhep.2016.07.020
39. Rao N, Rizk EM, Hwang R, et al. Nivolumab for hepatocellular carcinoma (HCC) in a real-world context. J Clin Oncol. 2019;37 (15_suppl):e15675. doi:10.1200/JCO.2019.37.15_suppl.e15675

40. Bristol-Myers Squibb Announces Results from CheckMate 459 study evaluating Opdivo (nivolumab) as a first-line treatment for patients with unresectable hepatocellular carcinoma [press release]. Available from: https://news.bms.com/press-release/bmy/bristol-myers-squibbannounces-results-checkmate-459-study-evaluating-opdivo-nivol: Bristol-MyersSquibb. Accessed June, 2019.

41. Yau T, Kang Y-K, Kim T-Y, et al. Nivolumab (NIVO) + ipilimumab (IPI) combination therapy in patients (pts) with advanced hepatocellular carcinoma (aHCC): results from CheckMate 040. J Clin Oncol. 2019;37(15_suppl):4012.

42. Kaseb AO, Carmagnani Pestana R, Vence LM, et al. Randomized, open-label, perioperative phase II study evaluating nivolumab alone versus nivolumab plus ipilimumab in patients with resectable HCC. $J$ Clin Oncol. 2019;37(4_suppl):185.

43. A phase 3 Study of KEYTRUDA ${ }^{\circledR}$ (pembrolizumab) in previously treated patients with advanced hepatocellular carcinoma. Merck. [press release].

44. Harding JJ, Nandakumar S, Armenia J, et al. Prospective genotyping of hepatocellular carcinoma: clinical implications of next-generation sequencing for matching patients to targeted and immune therapies. Clin Cancer Res. 2019;25(7):2116-2126. doi:10.1158/1078-0432.CCR-18-2293

45. Munker S, De Toni EN. Use of checkpoint inhibitors in liver transplant recipients. United Eur Gastroenterol J. 2018;6(7):970-973. doi:10.1177/2050640618774631

46. Kuo JC, Lilly LB, Hogg D. Immune checkpoint inhibitor therapy in a liver transplant recipient with a rare subtype of melanoma: a case report and literature review. Melanoma Res. 2018;28(1):61-64. doi:10.1097/CMR.0000000000000410

47. Ranganath HA, Panella TJ. Administration of ipilimumab to a liver transplant recipient with unresectable metastatic melanoma. $J$ Immunother (Hagerstown, MD: 1997). 2015;38(5):211.

48. Park BH, Hwang T, Liu TC, et al. Use of a targeted oncolytic poxvirus, JX-594, in patients with refractory primary or metastatic liver cancer: a phase I trial. Lancet Oncol. 2008;9(6):533-542. doi:10.1016/S1470-2045(08)70107-4

49. Greten TF, Sangro B. Targets for immunotherapy of liver cancer. $J$ Hepatol. 2017. 68(1):157-166.

50. Bulliard Y, Jolicoeur R, Zhang J, Dranoff G, Wilson NS, Brogdon JL. OX40 engagement depletes intratumoral Tregs via activating FcgammaRs, leading to antitumor efficacy. Immunol Cell Biol. 2014;92(6):475-480. doi:10.1038/icb.2014.26

51. Guo Z, Wang X, Cheng D, Xia Z, Luan M, Zhang S. PD-1 blockade and $\mathrm{OX} 40$ triggering synergistically protects against tumor growth in a murine model of ovarian cancer. PLoS ONE. 2014;9(2):e89350.

52. Sakuishi K, Apetoh L, Sullivan JM, Blazar BR, Kuchroo VK, Anderson AC. Targeting Tim-3 and PD-1 pathways to reverse T cell exhaustion and restore anti-tumor immunity. $J$ Exp Med. 2010;207(10):2187-2194. doi:10.1084/jem.20100643

53. Ribas A, Shin DS, Zaretsky J, et al. PD-1 blockade expands intratumoral memory T cells. Cancer Immunol Res. 2016;4(3):194-203. doi:10.1158/2326-6066.CIR-15-0210
OncoTargets and Therapy

\section{Publish your work in this journal}

OncoTargets and Therapy is an international, peer-reviewed, open access journal focusing on the pathological basis of all cancers, potential targets for therapy and treatment protocols employed to improve the management of cancer patients. The journal also focuses on the impact of management programs and new therapeutic agents and protocols on patient perspectives such as quality of life, adherence and satisfaction. The manuscript management system is completely online and includes a very quick and fair peer-review system, which is all easy to use. Visit http://www.dovepress.com/ testimonials.php to read real quotes from published authors. 